INPLASY

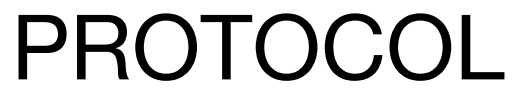

To cite: Wang et al. The efficacy and safety of Vortioxetine/Lu AA21004 in the treatment of major depressive disorder: a systematic review and meta-analysis. Inplasy protocol 202140011. doi: 10.37766/inplasy2021.4.0011

Received: 01 April 2021

Published: 02 April 2021

Corresponding author: Yi Wang

wang19831027@126.com

Author Affiliation:

Mental health center, Jiading

District, Shanghai

Support: Company.

Review Stage at time of this submission: Data analysis.

Conflicts of interest:

None declared.

\section{The efficacy and safety of Vortioxetine/Lu AA21004 in the treatment of major depressive disorder: a systematic review and meta-analysis}

Wang, Y1; Li, Z2; Shen, H³; Gao, H; Zhang, J5; Cheng, C6.

Review question / Objective: The efficacy and safety of Vortioxetine/Lu AA21004 in the treatment of major depressive disorder: a systematic review and meta-analysis.

Main outcome(s): Response (MADRS total scores decreased rated $\geq 50 \%$, HAMD-17/-24total score decreased rated $\geq 50 \%$, CGI-I score 1or 2).

Information sources: This study searched 5 databases of Pubmed, Embase, The Cochrane Library, Web of science and ClinicalTrails.gov. Search all literatures in the database before September 28, 2020. Search uses "Depression", "Vortioxetine" and "Randomize Controlled Trial" as subject words, and searches all their free words.

INPLASY registration number: This protocol was registered with the International Platform of Registered Systematic Review and Meta-Analysis Protocols (INPLASY) on 02 April 2021 and was last updated on 02 April 2021 (registration number INPLASY202140011).

\section{INTRODUCTION}

Review question / Objective: The efficacy and safety of Vortioxetine/Lu AA21004 in the treatment of major depressive disorder: a systematic review and meta-analysis.
Condition being studied: Depression is a common disease worldwide, with an estimated 350 million patients. Depression can lead to functional disability, a decline in quality of life and a reduction in life 
expectancy. According to World Health Organization (WHO) statistics, the number of people who commit suicide as a result of depression is estimated to be as high as 1 million every year, making it the second largest killer of human beings after cancer. The main treatment methods included drugs and psychologicals. Only about $30 \%$ of patients with depression can be cured, and less than $50 \%$ of patients with depression are effective with antidepressant drugs. At present, the main antidepressants are TCAs, SSRIs, SNRIs and NaSSA, etc. Vortioxetine/Lu AA21004. It is a new multimodal antidepressant jointly developed by Denmark's Lingbei Pharmaceutical (Lundbeck) and Japan's Takeda Pharmaceutical (Takeda). Trade name: Brintellix, Chemical name: 1-(2(2re4-dimethyl-phenylthio) phenylpiperazine, chemical structure formula: molecular formula: C18H22N2S fear HBR, molecular weight: 379.36 . It was approved for the treatment of depression by the FDA of the United States on September 30th, 2013. They are mainly 5-HT3 and 5-HT7 receptor antagonists, 5-HT1B receptor partial agonists, 5-HT1A receptor agonists and 5-HT transporter inhibitors. It can not only increase the content of $5-\mathrm{HT}$, but also enhance the sensitivity of the receptor to 5HT. However, it is not clear the optimal dose range of Vortioxetine/Lu AA21004 in the treatment of depression and the effect between different doses. Therefore, we systematically evaluated the efficacy and safety of different doses of Vortioxetine/Lu AA21004 in the treatment of adult depression by meta analysis, in order to provide reliable evidence-based basis for clinical application.

\section{METHODS}

Participant or population: Participants diagnosed with depression, of both sexes, of any ethnicity, and aged 18 years and older. The diagnostic criteria were DSMIV(APA 1994), DSM-IV-TR (APA 2000), DSM-5 (APA 2013), ICD-10(WHO 1992). All patients participated in the study voluntarily. We excluded studies of people with treatment-resistant depression, defined as inadequate treatment response to at least four weeks of adequate antidepressant treatment.

Intervention: Vortioxetine/Lu AA21004.

Comparator: Placebo or no treatment.

Study designs to be included: Randomized controlled trials (RCTs), single-blind or double-blind, placebo-control and only English literatures.

Eligibility criteria: Randomized controlled trials (RCTs), single-blind or double-blind, placebo-control and only English literatures.

Information sources: This study searched 5 databases of Pubmed, Embase, The Cochrane Library, Web of science and ClinicalTrails.gov. Search all literatures in the database before September 28, 2020. Search uses "Depression", "Vortioxetine" and "Randomize Controlled Trial" as subject words, and searches all their free words.

Main outcome(s): Response(MADRS total scores decreased rated $\geq 50 \%$ 、 HAMD-17/-24total score decreased rated $\geq 50 \%$, CGI-I score 1 or 2 ).

Additional outcome(s): 1. Remssion (MADRS total scores $\leq 10$, HAMD-17/-24 $\leq 7$, CGI-S score 1 or 2), Endpoint(6, 8 or 12 weeks MADRS total scores Mean \pm SD); 2. Adverse effects $\geq 5 \%$; 3 . Withdrawal rate: by calculating the proportion of the total number of people who dropped out during the study period in the full-analysis set.

Quality assessment / Risk of bias analysis: According to the method of RCT quality evaluation in Cochrane system evaluation manual 5.1.0, the methodological quality of the included literature was evaluated, including random method, allocation hiding, blind method, result evaluation, integrity of outcome data, selective reporting, and other sources of bias. The Gradeprofile 3.2.2 software was used to evaluate the evidence level of each analysis result, the reliability and accuracy of the result were observed. 
Strategy of data synthesis: The RevMan5.3 software provided by Cochrane collaboration network is used to analyze the extracted data by meta. Dichotomous data We analysed dichotomous data as risk ratios (RRs) with $95 \%$ confidence intervals (CI). We used the full-analysis set (FAS) database for efficacy analysis instead of ITT. We believed that not all shedding was due to the fact that non-responders or nonremitters, but also caused by imperfect baseline data or repetitive personnel. We thought that the full-analysis set (FAS) data was more scientific and reasonable. Continuous data We analysed continuous data as standardised mean difference (SMD) with $95 \% \mathrm{Cl}$. We entered and presented data with a consistent direction of effect. Data were analysed as endpoint data. We combined endpoint and change scores only if data were analysed as MD. If the literature contains the endpoints mean and standard deviation of the scale (MARDS, HAMD), it is extracted directly. If the endpoints mean and standard deviation of the scale are not shown in the literature, but the mean and standard deviation of the baseline period, the mean deviation and standard error of the baseline period and the sample size of the baseline period are provided, then the standard deviation of the endpoint need to be calculated. Analyses were conducted with the full-analysis set (FAS) data as reported (e.g. data from last observation carried forward (LOCF) or mixed model for repeated measurements (MMRM) methods).

Subgroup analysis: When there is significant heterogeneity, we will conduct a subgroup analysis of the different fixed dosing, different courses of treatment, and the race of the participants.

Sensitivity analysis: 1.Exclusion of studies that did not employ a double-blind approach; 2.Exclusion of studies with subsets of people with bipolar disorders; 3.Exclusion of trials with dropout rates of more than $20 \%$ in one of the treatment arms included; 4.Exclusion of studies with imputed data.
Country(ies) involved: China.

Keywords: Vortioxetine/Lu AA21004; Major Depressive Disorder.

Contributions of each author:

Author 1 - Yi Wang.

Author 2 - Zhibin Li.

Author 3 - Hongyan Shen.

Author 4 - Hongyan Gao.

Author 5 - Jing Zhang.

Author 6 - Cheng Cheng.

Language: English. 